\title{
Clinical Pearl: Words Matter: A Call for Re-Thinking SIDS
}

Melanie Wielicka, MD, PhD, Joseph R. Hageman, MD

"It appears, however, that since 1999, the incidence has remained fairly stable, with the most recent available data reporting 90.1 cases per 100,000. It is still estimated that about 3,400 infants die unexpectedly every year without an apparent cause [2]."

It has been over 50 years since Sudden Infant Death Syndrome (SIDS) was first described in 1969. It was initially defined simply as "the sudden death of any infant or young child, which is unexpected by history, and in which a thorough post mortem examination fails to demonstrate an adequate cause for death" [1]. Many studies have investigated the potential risk factors in an attempt to help establish ways to help decrease the incidence of unexplained infant death, and in fact, with the initiation of the American Academy of Pediatrics (AAP) safe sleep recommendations in 1992 (most recently updated in 2016) and the initiation of the Back To Sleep campaign in 1994, we observed a significant decline in SIDS cases all through the 90s [2, 3]. It appears, however, that since 1999, the incidence has remained fairly stable, with the most recent available data reporting 90.1 cases per 100,000 . It is still estimated that about 3,400 infants die unexpectedly every year without an apparent cause [2].

Since its original definition, several attempts have been made to characterize better and categorize what is known as sudden infant death syndrome. SIDS has remained the term most widely used by physicians. However, medical examiners and coroners have shifted away from using this term on death certificates, oftentimes replacing it with others, such as sudden unexplained infant death, death due to indeterminate cause, accidental suffocation, or strangulation in bed, or asphyxia $[4,5]$. Over the past years, it appears that one of the biggest controversies with regards to terminology has been related to the use of the word "syndrome." As this term refers to a group of symptoms occurring together or a medical condition encompassing a group of symptoms, many argue that it should not be used in reference to previously healthy infants with no alarming symptoms who died of an unexplained cause [5]. This debate and the resulting shift in diagnosis reporting can significantly affect epidemiological data through seemingly lower reported SIDS rates [4].

In a recently released clinical report, "Half Century Since SIDS: A Reappraisal of Terminology," the American Academy of Pediatrics calls for a consensus in nomenclature when describing those deaths [4]. The AAP points out the discrepancies can negatively impact the development of public health policies to prevent unexplained infant death. A lack of consistency in terminology when re- porting deaths affects research that relies on appropriately coded diagnoses for gathering data. It also makes it challenging to monitor mortality trends $[4,5]$ closely.

The report also describes in detail the history of terminology and classification related to unexpected infant death. It pays particular attention to the most recent definition from the 2017 National Association of Medical Examiners Panel on Sudden Infant Death in Pediatrics (NAME Panel), which included forensic pathologists representing NAME, pediatricians from the AAP, and federal liaisons from the CDC and NIH. They recommended using the term "unexplained sudden death," defined as "infant less than one year of age in apparent good health that dies suddenly and unexpectedly." They also suggested that deaths can then be further classified based on whether intrinsic (such as prematurity, febrile seizures) or extrinsic (non-lethal injuries or injuries of unknown significance) risk factors have been identified. There is also an additional set of criteria for accidental suffocation resulting in sleeprelated deaths.

\section{"They also suggested that deaths can then be further classified based on whether intrinsic (such as prematurity, febrile seizures) or extrinsic (non- lethal injuries or injuries of unknown significance) risk factors have been identified. There is also an additional set of criteria for accidental suffocation resulting in sleep-related deaths."}

A year later, a panel of physicians, forensic pathologists, epidemiologists, researchers, and parents, including some of the same experts included in the NAME panel, have met to discuss terminology related to sudden infant deaths, making recommendations to the World Health Organization for International Statistical Classification of Diseases and Related Health Problems, 11th revision. The current ICD coding includes three primary coding diagnoses: SIDS, unknown or unspecified cause, and accidental suffocation and strangulation in bed, all of which are included in a larger category of SUID (sudden unexplained infant death). The participants of the Radcliffe Congress recommended that sudden unexplained death in infancy and SIDS be classified under the same code. This recommendation was supported in the NAME Panel's publication in 2019, although the Panel did recommend using the term unexplained sudden death instead of SIDS [4].

Another concern with lack of consistency in terminology is how it could potentially affect communication with families [4, 6]. A wide range of terminology describing the same diagnosis can confuse families, lead to a more difficult bereavement process, and potentially lead to mistrust towards the medical system. A study that looked at the perception of diagnosis by parents of infants who 
died unexpectedly reported that families expressed dissatisfaction when they received inconsistent information from different healthcare professionals and frustration when a specific cause of death was not named [6]. In their report, the AAP emphasizes the importance of empathy and sensitivity when discussing sudden infant death with families. They also suggest that medical examiners develop a formal reporting mechanism to the primary care physicians, which could, in turn, discuss the investigation findings with the family. This mechanism could help facilitate an open discussion and help the physician provide appropriate referrals for specialists or mental health care. We should also remember that known risk factors for sudden infant death, such as smoking, unsafe sleeping environment, or prenatal exposure, are all public health issues. Per AAP recommendations, the presence of these risk factors alone should not lead to pressing legal charges against the family if child abuse has been eliminated [4].

\section{"In addition to being helpful from a health policy standpoint, this more consistent terminology will facilitate communication with families more effectively and objectively, creating better relationships and allowing us as physicians to better support grieving families."}

In their report, the AAP strongly supports the adaptation of the NAME Panel's terminology. They argue that it is clear and definitive, which will aid in gathering future epidemiological data and help guide future research on preventative measures. They also advocate changing the ICD classification and coding to that proposed by the Radcliffe Congress in 2018. In addition to being helpful from a health policy standpoint, this more consistent terminology will facilitate communication with families more effectively and objectively, creating better relationships and allowing us as physicians to better support grieving families.

As a personal note from someone who has worked in this area as an "apnea doc" and who has been an active member of the American Association of SIDS Prevention Physicians (AASPP) since its inception 30 years ago, I think SUID or SIDS still includes a heterogeneous group of etiologies for the sudden death of infants in the first year of life which, as we continue to search and find a new etiology, this group of infants will continue to get smaller. However, until we find all of the etiologies, we will need a code with a definition to categorize these infants and explain to their families and primary care physicians what we know and do not know about why each of these infants died. (Personal communication: JR Hageman, 10/8/2021).

\section{References:}

1. Beckwith JB. The sudden infant death syndrome. Curr Probl Pediatr. 1973 Jun;3(8):1-36. PMID: 4351768.

2. Center for Disease Control and Prevention. Sudden Unex- pected Infant Death and Sudden Infant Death Syndrome. Data and Statistics. Available at: https://www.cdc.gov/sids/ data.htm

3. Task Force on Sudden Infant Death Syndrome: Updated 2016 Recommendations for a Safe Infant Sleeping Environment. Pediatrics Nov 2016, 138 (5) e20162938; DOI: 10.1542/peds.2016-2938

4. Shapiro-Mendoza CK, Palusci VJ, Hoffman B, Batra E, Yester M, Corey TS, Sens MA; AAP TASK FORCE ON SUDDEN INFANT DEATH SYNDROME, COUNCIL ON CHILD ABUSE AND NEGLECT, COUNCIL ON INJURY, VIOLENCE, AND POISON PREVENTION, SECTION ON CHILD DEATH REVIEW AND PREVENTION, NATIONAL ASSOCIATION OF MEDICAL EXAMINERS. Half Century Since SIDS: A Reappraisal of Terminology. Pediatrics. 2021 Oct;148(4):e2021053746. doi: 10.1542/peds.2021-053746. Epub 2021 Sep 20. PMID: 34544849; PMCID: PMC8487943

5. Goldstein RD, Blair PS, Sens MA, et al. Inconsistent classification of unexplained sudden deaths in infants and children hinders surveillance, prevention and research: recommendations from The 3rd International Congress on Sudden Infant and Child Death. Forensic Sci Med Pathol. 2019;15(4):622628. doi:10.1007/s12024-019-00156-9

6. Crandall LG, Reno L, Himes B, Robinson D. The Diagnostic Shift of SIDS to Undetermined: Are There Unintended Consequences?. Acad Forensic Pathol. 2017;7(2):212-220. doi:10.23907/2017.022

Disclosures: Dr. Wielicka has nothing to disclose. Dr. Hageman is on the Advisory Board, Owlet, Inc.

NT
Clinical Pearls are published monthly.

Submission guidelines for "Clinical Pearls":

1250 word limit not including references or title page.

May begin with a brief case summary or example.

Summarize the pearl for emphasis.

No more than 7 references.

Please send your submissions to:

jhageman@peds.bsd.uchicago.edu

NEONATOLOGY TODAY is interested in publishing manuscripts from Neonatologists, Fellows, NNPs and those involved in caring for neonates on case studies, research results, hospital news, meeting announcements, and other pertinent topics.

Please submit your manuscript to: LomaLindaPublishingCompany@gmail.com 


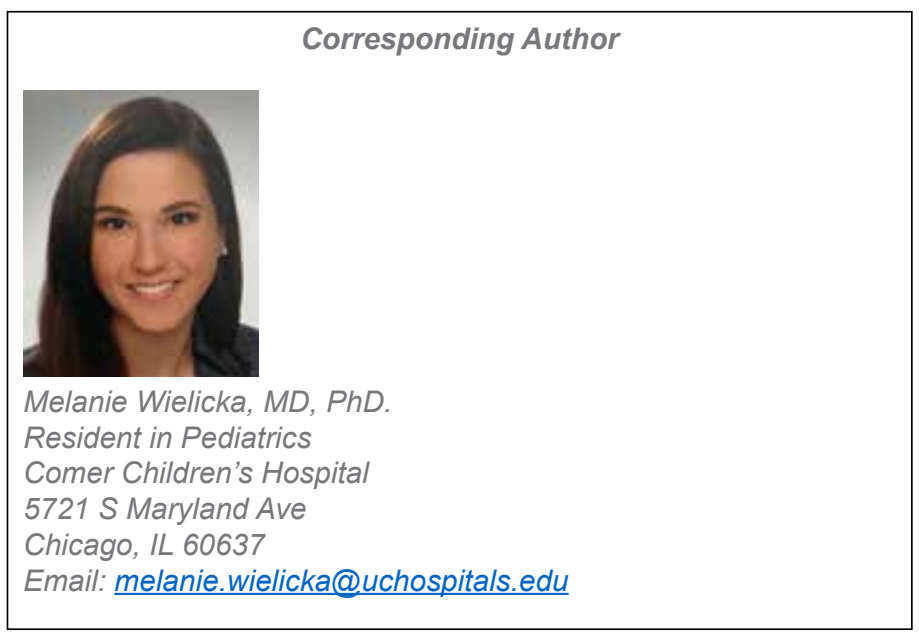

\section{Which Infants are More Vulnerable to Respiratory Syncytial Virus?}

RSV is a respiratory virus with cold-like symptoms that causes 90,000 hospitalizations and 4,500 deaths per year in children

5 and younger. It's 10 times more deadly than the flu.

For premature babies with fragile immune systems and underdeveloped lungs, RSV proves especially dangerous.

But risk factors associated with RSV don't touch all infants equally.*

\begin{tabular}{|c|c|c|}
\hline Caucasian Babies & Risk Factor & $\begin{array}{c}\text { African American } \\
\text { Babies }\end{array}$ \\
\hline $11.6 \%$ & Prematurity & $18.3 \%$ \\
\hline $58.1 \%$ & Breastfeeding & $50.2 \%$ \\
\hline $7.3 \%$ & $\begin{array}{l}\text { Low Birth } \\
\text { Weight }\end{array}$ & $11.8 \%$ \\
\hline $60.1 \%$ & Siblings & $71.6 \%$ \\
\hline $1 \%$ & $\begin{array}{l}\text { Crowded } \\
\text { Living } \\
\text { Conditions }\end{array}$ & $3 \%$ \\
\hline
\end{tabular}

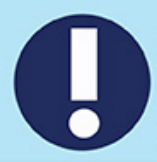

AFRICAN AMERICAN BABIES bear the brunt of RSV. Yet the American Academy of Pediatrics' restrictive new guidlines limit their access to RSV preventative treatment, increasing these babies' risk.

$$
\mathrm{A} f \mathrm{PA}
$$

Corresponding Author

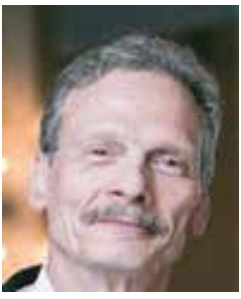

Joseph R. Hageman, MD

Senior Clinician Educator

Pritzker School of Medicine

University of Chicago

MC6060

5841 S. Maryland Ave.

Chicago, IL 60637

Phone: 773-702-7794

Fax: 773-732-0764

Email:jhageman@peds.bsd.uchicago.edu

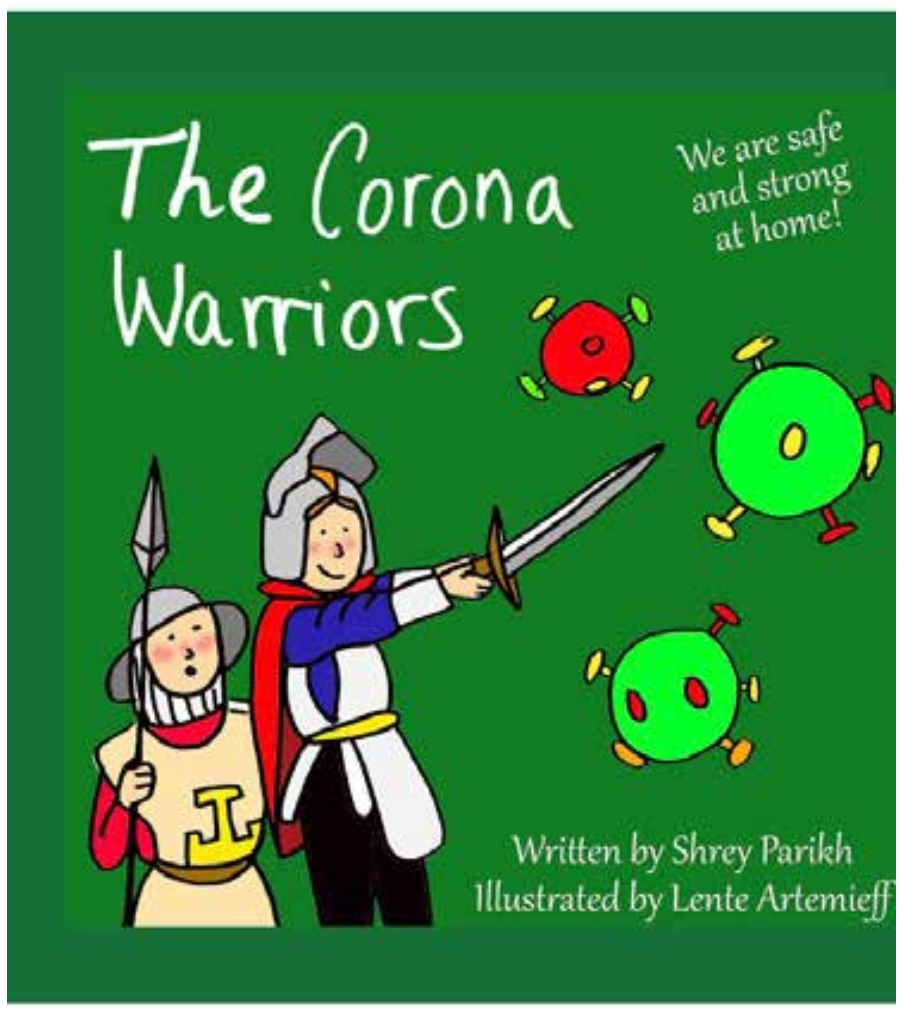

New subscribers are always welcome! NEONATOLOGY IQDAY

To sign up for free monthly subscription, just click on this box to go directly to our subscription page 